\title{
Call and Put Option Pricing with Discrete Linear Investment Strategy
}

\author{
Niloofar Ghorbani1 (), Andrzej Korzeniowski² \\ ${ }^{1}$ Department of Mathematical Sciences, High Point University, High Point, USA \\ ${ }^{2}$ Department of Mathematics, Arlington, Texas, USA \\ Email: nghorban@highpoint.edu; korzeiowski@uta.edu
}

How to cite this paper: Ghorbani, N. and Korzeniowski, A. (2022) Call and Put Option Pricing with Discrete Linear Investment Strategy. Journal of Mathematical Finance, 12, 84-96.

https://doi.org/10.4236/jmf.2022.121005

Received: November 29, 2021

Accepted: January 26, 2022

Published: January 29, 2022

Copyright $\odot 2022$ by author(s) and Scientific Research Publishing Inc. This work is licensed under the Creative Commons Attribution International License (CC BY 4.0).

http://creativecommons.org/licenses/by/4.0/

\begin{abstract}
We study the Option pricing with linear investment strategy based on discrete time trading of the underlying security, which unlike the existing continuous trading models, provides a feasible real market implementation. Closed form formulas for Call and Put Option price are established for fixed interest rates and their extensions to stochastic Vasicek and Hull-White interest rates.
\end{abstract}

\section{Keywords}

Discrete Dynamic Investment Strategy, Stochastic Interest Rates, Vasicek Model, Hull-White Model, European Call Option, European Put Option

\section{Introduction}

Financial derivatives market over the past few decades led to various generalizations of the classical Black-Scholes model. Notably, a seminal work of Wang et al. ([1] [2]) introduced a dynamic investment strategy in the underlying security for the purpose of hedging the investment risks. It turned out that selling a security proportionally to its dropping price for Put Option and buying the security proportionally to its rising price for Call Option (both under the Black-Scholes model) resulted in lower Option price. Zhang et al. [3] extended the result for Call Option to stochastic interest rates following the Vasicek model. Subsequently Ghorbani and Korzeniowski [4] and [5] obtained the Call Option price with investment strategy for the Cox-Ingersoll-Ross (CIR) interest rates and the HullWhite respectively.

A key assumption in the above modeling relies on the fact that the stock trading by the option holder is done in continuous time. To remedy this drawback 
and accommodate the actual market implementation, Meng Li et al. [6] proposed a discrete time investment strategy for Call Option pricing in the case of non-random constant interest rate and the stock price following the Geometric Fractional Brownian Motion (GFBM).

Novelty of dynamic investment strategies, and their discrete time market implementation presented here, is two-fold. Firstly, unlike in the classical BlackScholes models where the investor buys options and has no position in the underlying stock throughout the option time horizon, the dynamic investment strategy requires the investor to trade the stock at discrete times associated with the stock price crossing certain levels in the pre-determined price range, whereby lowering the investor's risk, manifested by the lower option price. Secondly, the interest rates are no longer constant and are assumed stochastic.

In this paper the stock price follows the Geometric Brownian Motion (GBM). We first extend the discrete investment strategy for Call Option [6] to random interest rates under the Vasicek and Hull-White models, and subsequently we derive European Put Option price for non-random constant interest rate followed by Vasicek and Hull-White stochastic interest rates respectively.

The remainder of the paper is organized as follows. Section 2 describes the Call Option assumptions and Value function in a discrete dynamic model. Option Value under both fixed interest rate and stochastic interest rate for Vasicek and Hull-White models has been derived. In Section 3, the Put Option assumptions, Value function and price under fixed and stochastic interest rate models are presented. The last section concludes the paper.

\section{Call Option}

The adaptive risk hedging for European Call Option is proposed based on the following assumptions [6]:

- The Call Option holder holds one Option contract and the initial Capital of amount $A=Q \times K$ at the beginning of the Option period, where $Q$ is the number of shares of stock for Option contract and $K$ is the Call Option strike price.

- The Call Option holder should buy the underlying stock according to the price changes subject to the discrete trading strategy.

- The potential loss sustained by the Option holder through adaptive trading is not the responsibility of the Option writer.

- There are no transaction costs for buying the underlying stock.

\subsection{Description of Discrete Trading Strategy}

The Call Option contains a capital amount $A$ initially. When the price of the underlying stock goes up to $(K+\delta), \delta \geq 0$ the Option holder spends $\beta_{0} A$ to buy stocks. The parameter $\beta_{0}$ is a constant between 0 and 1 , called the initial capital utilization coefficient. The Option holder linearly adjusts the capital utilization to increase the holding while the price continues to increase and hits a 
series of equally spaced points $S_{n}$ where $\left\{S_{n}: S_{n}=K-n \Delta, n=1,2, \cdots, N\right\}$, with $\Delta$ a positive constant, the price distance for two consecutive trading actions and $N$ the total number of trades within the capital valid period.

The strategy parameters $\alpha$ (investment index) and $\beta$ (minimum capital utilization), both positive numbers, illustrate the maximum amount of capital tradable is $\beta A$ when the price reaches $(1+\alpha)(K+\delta)$. The strategy assumes the Option holder will evenly $\left(\frac{\beta A}{N}\right)$ distribute the capital over each of the potential trades corresponding to $S_{n}, n=1,2, \cdots, N$ by selling $\frac{\beta A}{N S_{n}}$ shares of stock [6].

\subsection{Value Function}

It was found in [6] that the Call Option value $V_{T}$ based on the discrete trading strategy reads

$$
V_{T}=\left\{\begin{array}{lc}
0 & S_{T}<K \\
S_{T}-K & K \leq S_{T}<S_{1} \\
S_{T}-\frac{\beta S_{T} K}{N} \sum_{n=1}^{m} \frac{1}{K+n \Delta}+\left(\frac{\beta m}{N}-1\right) K & S_{m} \leq S_{T}<S_{m+1} \leq S_{N} \\
S_{T}-\frac{\beta S_{T} K}{N} \sum_{n=1}^{N} \frac{1}{K+n \Delta}+(\beta-1) K & S_{N} \leq S_{T}
\end{array}\right.
$$

with the investment parameters $\alpha, \beta$, strike price $K$ and the terminal stock price $S_{T}$ where,

$\alpha$ is the investment strategy index, indicating the stock investment occurs during the period in which the stock price increases from $K$ to $(1+\alpha) K$.

$\beta$ is the maximum value of the stock investment proportion.

Furthermore, based on (2.1), the Call Option pricing formula for fixed interest rate and the stock price following the geometric fractional Brownian Motion was presented in [6]. In what follows, we derive the Call Option price for the discrete time investment strategy for non-random, Vasicek, and Hull-White interest rate models.

\subsection{Option Price under Fixed Interest Rates}

Assume the dynamic of the stock price $S_{T}$ under the risk-neutral measure follows

$$
\mathrm{d} S_{t}=r S_{t} \mathrm{~d} t+\sigma_{1} S_{t} \mathrm{~d} W_{1, t}, \quad S(0)=S_{0}>0,0 \leq t \leq T .
$$

By Ito formula, the stock price at the maturity time $T$ can be obtained and reads

$$
S_{T}=S_{0} \mathrm{e}^{\left[\left(r-\frac{1}{2} \sigma_{1}^{2}\right) t+\sigma_{1} W_{1, T}\right]} .
$$

Based on the value function $V_{T}$ in (2.1), the Call Option price under riskneutral measure can be evaluated as 


$$
C=\mathrm{e}^{-r T} E\left[V_{T}\right]
$$

where,

$$
\begin{aligned}
E\left[V_{T}\right]= & \int_{K}^{S_{1}}\left(S_{T}-K\right) f\left(S_{T}\right) \mathrm{d} S_{T} \\
& +\int_{S_{m}}^{S_{m+1}}\left[\left(1-\frac{\beta K}{N} \sum_{n=1}^{m} \frac{1}{K+n \Delta}\right) S_{T}+\left(\frac{\beta m}{N}-1\right) K\right] f\left(S_{T}\right) \mathrm{d} S_{T} \\
& +\int_{S_{N}}^{+\infty}\left[\left(1-\frac{\beta K}{N} \sum_{n=1}^{N} \frac{1}{K+n \Delta}\right) S_{T}+(\beta-1) K\right] f\left(S_{T}\right) \mathrm{d} S_{T}
\end{aligned}
$$

with

$$
\begin{aligned}
& I_{1}=\int_{K}^{S_{1}}\left(S_{T}-K\right) f\left(S_{T}\right) \mathrm{d} S_{T} \\
& I_{2}=\int_{S_{m}}^{S_{m+1}}\left[\left(1-\frac{\beta K}{N} \sum_{n=1}^{m} \frac{1}{K+n \Delta}\right) S_{T}+\left(\frac{\beta m}{N}-1\right) K\right] f\left(S_{T}\right) \mathrm{d} S_{T} \\
& I_{3}=\int_{S_{N}}^{+\infty}\left[\left(1-\frac{\beta K}{N} \sum_{n=1}^{N} \frac{1}{K+n \Delta}\right) S_{T}+(\beta-1) K\right] f\left(S_{T}\right) \mathrm{d} S_{T}
\end{aligned}
$$

Calculating the three integrals $I_{1}, I_{2}$ and $I_{3}$ with a similar method to the derivations in [2] and [3]. Using the probability density function of $\ln S_{T}$, [7] [8] [9] with mean and variance as follow

$$
\begin{gathered}
\mu=E\left[\ln S_{T}\right]=E\left[\ln S_{0}+\left(r-\frac{1}{2} \sigma_{1}^{2}\right) T+\sigma_{1} W_{1, T}\right]=\ln S_{0}+\left(r-\frac{1}{2} \sigma_{1}^{2}\right) T \\
\sigma_{1}^{2}=\operatorname{Var}\left[\ln S_{0}+\left(r-\frac{1}{2} \sigma_{1}^{2}\right) T+\sigma_{1} W_{1, T}\right]=\sigma_{1}^{2} T
\end{gathered}
$$

gives the Call Option price.

$$
\begin{aligned}
& I_{1}=\int_{K}^{S_{1}}\left(S_{T}-K\right) f\left(S_{T}\right) \mathrm{d} S_{T} \\
& =\int_{\ln K}^{\ln S_{1}}\left(\mathrm{e}^{y}-K\right) f\left(\mathrm{e}^{y}\right) \mathrm{e}^{y} \mathrm{~d} y \\
& =\int_{\ln K}^{\ln S_{1}}\left(\mathrm{e}^{y}-K\right) \frac{1}{\sqrt{2 \pi} \sigma} \mathrm{e}^{-\frac{1}{2} \frac{(y-\mu)^{2}}{\sigma^{2}}} \mathrm{~d} y \\
& =\int_{\ln K}^{\ln S_{1}} \frac{1}{\sqrt{2 \pi} \sigma} \mathrm{e}^{y} \mathrm{e}^{-\frac{1}{2} \frac{(y-\mu)^{2}}{\sigma^{2}}} \mathrm{~d} y-K \int_{\ln K}^{\ln S_{1}} \frac{1}{\sqrt{2 \pi} \sigma} \mathrm{e}^{-\frac{1}{2} \frac{(y-\mu)^{2}}{\sigma^{2}}} \mathrm{~d} y \\
& =\int_{\frac{\ln K-\mu}{\sigma}}^{\frac{\ln S_{1}-\mu}{\sigma}} \frac{1}{\sqrt{2 \pi}} \mathrm{e}^{\mu+\sigma z} \mathrm{e}^{-\frac{1}{2} z^{2}} \mathrm{~d} z-K \int_{\frac{\ln K-\mu}{\sigma}}^{\frac{\ln S_{1}-\mu}{\sigma}} \frac{1}{\sqrt{2 \pi}} \mathrm{e}^{-\frac{1}{2} z^{2}} \mathrm{~d} z \\
& =\frac{1}{\sqrt{2 \pi}} \int_{\frac{\ln K-\mu}{\sigma}}^{\frac{\ln S_{1}-\mu}{\sigma}} \mathrm{e}^{\mu+\frac{1}{2} \sigma_{2}} \mathrm{e}^{-\frac{1}{2}(z-\sigma)^{2}} \mathrm{~d} z-K \frac{1}{\sqrt{2 \pi}} \int_{\frac{\ln K-\mu}{\sigma}}^{\frac{\ln S_{1}-\mu}{\sigma}} \mathrm{e}^{-\frac{1}{2} z^{2}} \mathrm{~d} z \\
& =\mathrm{e}^{\mu+\frac{1}{2} \sigma_{2}}\left[N\left(\frac{\ln S_{1}-\mu-\sigma^{2}}{\sigma}\right)-N\left(\frac{\ln K-\mu-\sigma^{2}}{\sigma}\right)\right] \\
& -K\left[N\left(\frac{\ln S_{1}-\mu}{\sigma}\right)-N\left(\frac{\ln K-\mu}{\sigma}\right)\right]
\end{aligned}
$$

and 


$$
\begin{aligned}
& I_{2}=\int_{S_{m}}^{S_{m+1}}\left[\left(1-\frac{\beta K}{N} \sum_{n=1}^{m} \frac{1}{K+n \Delta}\right) S_{T}+\left(\frac{\beta m}{N}-1\right) K\right] f\left(S_{T}\right) \mathrm{d} S_{T} \\
& =\int_{S_{m}}^{S_{m+1}}\left(1-\frac{\beta K}{N} \sum_{n=1}^{m} \frac{1}{K+n \Delta}\right) S_{T} f\left(S_{T}\right) \mathrm{d} S_{T}+\int_{S_{m}}^{S_{m+1}}\left(\frac{\beta m}{N}-1\right) K f\left(S_{T}\right) \mathrm{d} S_{T} \\
& =\left(1-\frac{\beta K}{N} \sum_{n=1}^{m} \frac{1}{K+n \Delta}\right) \int_{S_{m}}^{S_{m+1}} \mathrm{e}^{y} f\left(\mathrm{e}^{y}\right) \mathrm{e}^{y} \mathrm{~d} y+\left(\frac{\beta m}{N}-1\right) K \int_{S_{m}}^{S_{m+1}} f\left(\mathrm{e}^{y}\right) \mathrm{e}^{y} \mathrm{~d} y \\
& =\frac{1}{\sqrt{2 \pi} \sigma}\left(1-\frac{\beta K}{N} \sum_{n=1}^{m} \frac{1}{K+n \Delta}\right) \int_{S_{m}}^{S_{m+1}} \mathrm{e}^{y} \mathrm{e}^{-\frac{1}{2}} \frac{(y-\mu)^{2}}{\sigma^{2}} \mathrm{~d} y \\
& +\frac{1}{\sqrt{2 \pi} \sigma}\left(\frac{\beta m}{N}-1\right) K \int_{S_{m}}^{S_{m+1}} \mathrm{e}^{-\frac{1}{2}} \frac{(y-\mu)^{2}}{\sigma^{2}} \mathrm{~d} y \\
& =\frac{1}{2 \pi}\left(1-\frac{\beta K}{N} \sum_{n=1}^{m} \frac{1}{K+n \Delta}\right) \frac{\int_{\frac{S_{m+1}-\mu}{\sigma}}^{\sigma}}{\sigma} \mathrm{e}^{\mu+z \sigma} \mathrm{e}^{-\frac{1}{2} z^{2}} \mathrm{~d} z \\
& +\frac{1}{2 \pi}\left(\frac{\beta m}{N}-1\right) K \int_{\frac{S_{m}-\mu}{\sigma}}^{\frac{S_{m+1}-\mu}{\sigma}} \mathrm{e}^{-\frac{1}{2} z^{2}} \mathrm{~d} z \\
& =\mathrm{e}^{\mu+\frac{1}{2} \sigma^{2}}\left(1-\frac{\beta K}{N} \sum_{n=1}^{m} \frac{1}{K+n \Delta}\right)\left[N\left(\frac{\ln S_{m+1}-\mu-\sigma^{2}}{\sigma}\right)-N\left(\frac{\ln S_{m}-\mu-\sigma^{2}}{\sigma}\right)\right] \\
& +\left(\frac{\beta m}{N}-1\right) K\left[N\left(\frac{\ln S_{m+1}-\mu}{\sigma}\right)-N\left(\frac{\ln S_{m}-\mu}{\sigma}\right)\right]
\end{aligned}
$$

Thus,

$$
\begin{aligned}
I_{3}= & \int_{S_{N}}^{+\infty}\left[\left(1-\frac{\beta K}{N} \sum_{n=1}^{N} \frac{1}{K+n \Delta}\right) S_{T}+(\beta-1) K\right] f\left(S_{T}\right) \mathrm{d} S_{T} \\
= & \left(1-\frac{\beta K}{N} \sum_{n=1}^{N} \frac{1}{K+n \Delta}\right) \int_{\ln S_{N}}^{+\infty} \mathrm{e}^{y} f\left(\mathrm{e}^{y}\right) \mathrm{e}^{y} \mathrm{~d} y+(\beta-1) K \int_{\ln S_{N}}^{+\infty} f\left(\mathrm{e}^{y}\right) \mathrm{e}^{y} \mathrm{~d} y \\
= & \left(1-\frac{\beta K}{N} \sum_{n=1}^{N} \frac{1}{K+n \Delta}\right) \frac{1}{\sqrt{2 \pi} \sigma} \int_{\ln S_{N}}^{+\infty} \mathrm{e}^{y} \mathrm{e}^{-\frac{1}{2}} \frac{(y-\mu)^{2}}{\sigma^{2}} \mathrm{~d} y \\
& +(\beta-1) K \frac{1}{\sqrt{2 \pi} \sigma} \int_{\ln S_{N}}^{+\infty} \mathrm{e}^{-\frac{1}{2}} \frac{(y-\mu)^{2}}{\sigma^{2}} \mathrm{~d} y \\
= & \mathrm{e}^{\mu+\frac{1}{2} \sigma^{2}}\left(1-\frac{\beta K}{N} \sum_{n=1}^{N} \frac{1}{K+n \Delta}\right)\left[N\left(\frac{\mu+\sigma^{2}-\ln S_{N}}{\sigma}\right)\right] \\
& +(\beta-1) K N\left(\frac{\mu-\ln S_{N}}{\sigma}\right)
\end{aligned}
$$

By combining (2.4) to (2.11), the Option Price under fixed interest rate reads

$$
\begin{aligned}
C= & \mathrm{e}^{-r T} E\left[V_{T}\right] \\
= & \mathrm{e}^{-r T} \mathrm{e}^{\mu+\frac{1}{2} \sigma^{2}}\left[N\left(d_{1}\right)-N\left(d_{2}\right)\right]-K \mathrm{e}^{-r T}\left[N\left(d_{3}\right)-N\left(d_{4}\right)\right] \\
& +\mathrm{e}^{-r T} \mathrm{e}^{\mu+\frac{1}{2} \sigma^{2}}\left(1-\frac{\beta K}{N} \sum_{n=1}^{m} \frac{1}{K+n \Delta}\right)\left[N\left(d_{5}\right)-N\left(d_{6}\right)\right] \\
& +\mathrm{e}^{-r T}\left(\frac{\beta m}{N}-1\right)\left[N\left(d_{7}\right)-N\left(d_{8}\right)\right] \\
& +\mathrm{e}^{-r T} \mathrm{e}^{\mu+\frac{1}{2} \sigma^{2}}\left(1-\frac{\beta K}{N} \sum_{n=1}^{m} \frac{1}{K+n \Delta}\right) N\left(d_{9}\right)+\mathrm{e}^{-r T} K(\beta-1) N\left(d_{10}\right)
\end{aligned}
$$


where,

$$
\begin{array}{ll}
d_{1}=\frac{\ln S_{1}-\mu-\sigma^{2}}{\sigma} & d_{6}=\frac{\ln S_{m}-\mu-\sigma^{2}}{\sigma} \\
d_{2}=\frac{\ln K-\mu-\sigma^{2}}{\sigma} & d_{7}=\frac{\ln S_{m+1}-\mu}{\sigma} \\
d_{3}=\frac{\ln S_{1}-\mu-\sigma^{2}}{\sigma} & d_{8}=\frac{\ln S_{m}-\mu}{\sigma} \\
d_{4}=\frac{\ln K-\mu}{\sigma} & d_{9}=\frac{\mu+\sigma^{2}-\ln S_{N}}{\sigma} \\
d_{5}=\frac{\ln S_{m+1}-\mu-\sigma^{2}}{\sigma} & d_{10}=\frac{\mu-\ln S_{N}}{\sigma}
\end{array}
$$

and

$$
\begin{aligned}
& \mu=\ln S_{0}+\left(\mu-\frac{1}{2} \sigma_{1}^{2}\right) T \\
& \sigma^{2}=\sigma_{1}^{2} T .
\end{aligned}
$$

\subsection{Option Price under Stochastic Interest Rates}

\subsubsection{Vasicek Model}

Solution to the Vasicek interest rate model [10] can be used to obtain the Call Option price $C$ according to the following formula

$$
C=P(0, T) E^{T}\left[V_{T}\right]
$$

where

$$
S_{T}=S_{0} \mathrm{e}^{\int_{0}^{T}\left(r_{s}-\frac{1}{2} \sigma_{1}^{2}\right) \mathrm{ds}+\int_{0}^{T} \sigma_{1} \mathrm{~d} W_{1, t}}
$$

and $P(0, T)$ is the price of the zero-coupon bond. The mean $\mu_{T}$ and variance $\sigma_{T}^{2}$ in the case of Vasicek model were obtained under the $T$-forward measure by Zhang [3]. Therefore, the expectation of $V_{T}$ under the $T$-forward measure for the discrete dynamic strategy can be obtained similarly the derivation of (2.12) and gives

$$
\begin{aligned}
C= & P(0, T) \mathrm{e}^{\mu_{T}+\frac{1}{2} \sigma_{T}^{2}}\left[N\left(d_{1}\right)-N\left(d_{2}\right)\right]-K P(0, T)\left[N\left(d_{3}\right)-N\left(d_{4}\right)\right] \\
& +P(0, T) \mathrm{e}^{\mu_{T}+\frac{1}{2} \sigma_{T}^{2}}\left(1-\frac{\beta K}{N} \sum_{n=1}^{m} \frac{1}{K+n \Delta}\right)\left[N\left(d_{5}\right)-N\left(d_{6}\right)\right] \\
& +P(0, T)\left(\frac{\beta m}{N}-1\right)\left[N\left(d_{7}\right)-N\left(d_{8}\right)\right] \\
& +P(0, T) \mathrm{e}^{\mu_{T}+\frac{1}{2} \sigma_{T}^{2}}\left(1-\frac{\beta K}{N} \sum_{n=1}^{m} \frac{1}{K+n \Delta}\right) N\left(d_{9}\right) \\
& +P(0, T) K(\beta-1) N\left(d_{10}\right)
\end{aligned}
$$

where

$$
\begin{array}{ll}
d_{1}=\frac{\ln S_{1}-\mu_{T}-\sigma_{T}^{2}}{\sigma_{T}} & d_{6}=\frac{\ln S_{m}-\mu_{T}-\sigma_{T}^{2}}{\sigma_{T}} \\
d_{2}=\frac{\ln K-\mu_{T}-\sigma_{T}^{2}}{\sigma_{T}} & d_{7}=\frac{\ln S_{m+1}-\mu_{T}}{\sigma_{T}}
\end{array}
$$




$$
\begin{array}{ll}
d_{3}=\frac{\ln S_{1}-\mu_{T}-\sigma_{T}^{2}}{\sigma_{T}} & d_{8}=\frac{\ln S_{m}-\mu_{T}}{\sigma_{T}} \\
d_{4}=\frac{\ln K-\mu_{T}}{\sigma_{T}} & d_{9}=\frac{\mu_{T}+\sigma^{2}-\ln S_{N}}{\sigma_{T}} \\
d_{5}=\frac{\ln S_{m+1}-\mu_{T}-\sigma_{T}^{2}}{\sigma_{T}} & d_{10}=\frac{\mu_{T}-\ln S_{N}}{\sigma_{T}}
\end{array}
$$

and

$$
\begin{aligned}
& \mu_{T}=\ln S_{0}-\frac{\sigma_{1}^{2}}{2} T-r_{0} \frac{1-\mathrm{e}^{-a T}}{a}+\left(\frac{\theta}{a}-\frac{\sigma_{2}^{2}}{a^{2}}\right)\left[T-\frac{1-\mathrm{e}^{-a T}}{a}\right]+\frac{\sigma_{2}^{2}}{2 a} \frac{1-\mathrm{e}^{-a T}}{a} \\
& \sigma_{T}^{2}=\frac{\sigma_{2}^{2}}{a^{2}}\left[T-2 \frac{1-\mathrm{e}^{-a T}}{a}+\frac{1-\mathrm{e}^{-2 a T}}{2 a}\right]+\sigma_{1}^{2} T
\end{aligned}
$$

with the zero-coupon bond price

$$
P(0, T)=\exp \left\{\left[\frac{1-\mathrm{e}^{-a T}}{a}-T\right]\left(\frac{\theta}{a}-\frac{\sigma_{2}^{2}}{2 a^{2}}\right)-\frac{\sigma_{2}^{2} \frac{1-\mathrm{e}^{-a T}}{a}}{4 a}\right\} \mathrm{e}^{-r_{0} \frac{1-\mathrm{e}^{-a T}}{a}} .
$$

\subsubsection{Hull-White Model}

We derived the Option price under Hull-White interest rates model [4] via solving Hull-White $S D E$. Since our derivation is under $T$-forward measure, we need to consider the zero-coupon bond price $P(0, T)$ and the mean $\mu_{T}$ and Variance $\sigma_{T}^{2}$ for this model under the $T$-forward measure. The Option price in this case is expressed by (2.15), where

$$
\begin{aligned}
\mu_{T}= & \ln S_{0}-\frac{\sigma_{1}^{2}}{2} T+r_{0} \frac{1-\mathrm{e}^{-a T}}{a}-\frac{\sigma_{2}^{2}}{a}\left[\frac{\mathrm{e}^{-2 a T}\left((2 a T-3) \mathrm{e}^{2 a T}+4 \mathrm{e}^{a T}-1\right)}{2 a^{2}}\right] \\
& +\int_{0}^{T} \mathrm{e}^{-a t} \int_{0}^{t} \theta(s) \mathrm{e}^{a s} \mathrm{~d} s \mathrm{~d} t \\
\sigma_{T}^{2}= & \frac{\sigma_{2}^{2}}{a^{2}}\left[T-2 \frac{1-\mathrm{e}^{-a T}}{a}+\frac{1-\mathrm{e}^{-2 a T}}{2 a}\right]+\sigma_{1}^{2} T
\end{aligned}
$$

and the zero-coupon bond price is

$$
P(0, T)=\mathrm{e}^{\frac{r_{0}\left(\mathrm{e}^{-a T}-1\right)}{a}-\int_{0}^{T} \mathrm{e}^{-a s} \int_{0}^{5} \theta(u) \mathrm{e}^{a u} \mathrm{duds}+\frac{\sigma_{2}^{2}}{2 a^{2}}\left[T+\frac{1-\mathrm{e}^{-2 a T}}{2 a}-\frac{2}{a}\left(1-\mathrm{e}^{-a T}\right)\right]} .
$$

\section{Put Options}

The Put Option contains a specified capital amount $A$ and holding of $Q$ initially. When the stock price drops from $K$ to $(K-\delta), \delta \geq 0$ the Option holder sells $\beta_{0} A$ proportion of stocks. Parameter $\beta_{0}$ is called the initial capital utilization coefficient which is a constant between 0 and 1 . The Option holder linearly adjusts the capital utilization to decrease the holding if the price continues to fall until it reaches $(1-\alpha)(K-\delta)$ and the total capital spending reaches $\beta A$ with $\beta<\cdots<\beta_{1}<\beta_{0}$. The Option holder will only sell when the stock price 
hits a series of equally spaced points $S_{n}$, where $\left\{S_{n}: S_{n}=K-n \Delta, n=1,2, \cdots, N\right\}$, with $\Delta$ a positive constant, the price distance for two consecutive trading actions and $N$ the total number of trades within the capital valid period. The strategy parameters $\alpha$ (investment index) and $\beta$ (minimum capital utilization), both positive numbers, illustrate the maximum amount of capital tradable is $\beta_{0} A$ when the price reaches $(1-\alpha)(K-\delta)$. The strategy assumes the Option holder will evenly $\left(\frac{\beta A}{N}\right)$ distribute the capital over each of the potential trades corresponding to $S_{n}, n=1,2, \cdots, N$ by selling $\frac{\beta A}{N S_{n}}$ shares of stock.

\subsection{Value Function}

In the classical European Options, the Option writer will sustain a loss of the amount $L$ as the stock price falls below $K$

$$
L=Q(K-S)=\frac{A}{K}(K-S) .
$$

To adaptive hedging Option, the investor is required to sell a proportion of underlying stock in order to hedge the risk. In a discrete position strategy, the Option holder's income $R$ based on such transactions throughout the Option valid period can be calculated as,

$$
R=\frac{\beta A}{N S_{n}}\left(S_{n}-S\right)
$$

when the stock price falls from $S_{n}$ to $S$, with $S_{N} \leq S_{m+1}<S<S_{n}$.

The Option holder makes a cumulative income $R(S)$ as

$$
R(S)=\sum_{n=1}^{m} \frac{\beta A}{N S_{n}}\left(S_{n}-S\right)
$$

Thus, the total loss taken by the Option writer can be obtained as

$$
\begin{aligned}
L(S) & =\frac{A}{K}(K-S)-\sum_{n=1}^{m} \frac{\beta A}{N S_{n}}\left(S_{n}-S\right) \\
& =\frac{A}{K}(K-S)-\frac{\beta A}{N} \sum_{n=1}^{m} \frac{\left(S_{n}-S\right)}{S_{n}} \\
& =\frac{A}{K}(K-S)-\frac{\beta A}{N} \sum_{n=1}^{m} \frac{S_{n}}{S_{n}}+\frac{\beta A S}{N} \sum_{n=1}^{m} \frac{1}{S_{n}} \\
& =A-\frac{A S}{K}-\frac{\beta A m}{N}+\frac{\beta A S}{N} \sum_{n=1}^{m} \frac{1}{K-n \Delta} \\
& =\left(1-\frac{\beta m}{N}\right) A-\frac{A S}{K}+\frac{\beta A S}{N} \sum_{n=1}^{m} \frac{1}{K-n \Delta}
\end{aligned}
$$

however, for $S \leq S_{N}$, the Option writer's loss $L$ is

$$
L(S)=(1-\beta) A-\frac{A S}{K}+\frac{\beta A S}{N} \sum_{n=1}^{m} \frac{1}{K-n \Delta} .
$$

Therefore, the following function represents the Option writer's Loss based on 
the stock price $S$

$$
L(S)=\left\{\begin{array}{lc}
(1-\beta) A-\frac{A S}{K}+\frac{\beta A S}{N} \sum_{n=1}^{m} \frac{1}{K-n \Delta} & S \leq S_{N} \\
\left(1-\frac{\beta m}{N}\right) A-\frac{A S}{K}+\frac{\beta A S}{N} \sum_{n=1}^{m} \frac{1}{K-n \Delta} & S_{N} \leq S_{m+1}<S \leq S_{m} \\
\frac{A}{K}(K-S) & S_{1}<S \leq K \\
0 & S>K
\end{array}\right.
$$

Consequently, the intrinsic value function $V(S)$ is derived by dividing the loss function by the number of shares of stock within the Option depending on the stock finishing price $S_{T}$

$$
V\left(S_{T}\right)=\left\{\begin{array}{lc}
(1-\beta) K-S_{T}+\frac{\beta S_{T} K}{N} \sum_{n=1}^{m} \frac{1}{K-n \Delta} & S_{T} \leq S_{N} \\
\left(1-\frac{\beta m}{N}\right) K-S_{T}+\frac{\beta S_{T} K}{N} \sum_{n=1}^{m} \frac{1}{K-n \Delta} & S_{N} \leq S_{m+1}<S_{T} \leq S_{m} \\
K-S_{T} & S_{1}<S_{T} \leq K \\
0 & S_{T}>K
\end{array}\right.
$$

\subsection{Option Price under Fixed Interest Rates}

Considering the assumptions of section (3.1) and the intrinsic value function $V\left(S_{T}\right)$ (3.7), the Put Option price under risk-neutral measure is evaluated as

$$
P=\mathrm{e}^{-r T} E\left[V\left(S_{T}\right)\right]
$$

where,

$$
\begin{aligned}
E\left[V\left(S_{T}\right)\right]= & \int_{0}^{S_{N}}\left[(1-\beta) K-S_{T}+\frac{\beta S_{T} K}{N} \sum_{n=1}^{m} \frac{1}{K-n \Delta}\right] f\left(S_{T}\right) \mathrm{d} S_{T} \\
& +\int_{S_{m+1}}^{S_{m}}\left[\left(1-\frac{\beta m}{N}\right) K-S_{T}+\frac{\beta S_{T} K}{N} \sum_{n=1}^{m} \frac{1}{K-n \Delta}\right] f\left(S_{T}\right) \mathrm{d} S_{T} \\
& +\int_{S_{1}}^{K}\left(K-S_{T}\right) f\left(S_{T}\right) \mathrm{d} S_{T}
\end{aligned}
$$

calculating the three integrals $I_{1}, I_{2}$ and $I_{3}$ similar to the previous sections and using the probability density function of $\ln S_{T}$, whose mean and variance has been obtained in (2.7) and (2.8) gives the Put Option price.

$$
\begin{aligned}
I_{1}= & \int_{0}^{S_{N}}\left[(1-\beta) K-S_{T}+\frac{\beta S_{T} K}{N} \sum_{n=1}^{m} \frac{1}{K-n \Delta}\right] f\left(S_{T}\right) \mathrm{d} S_{T} \\
= & \int_{0}^{S_{N}}(1-\beta) K f\left(S_{T}\right) \mathrm{d} S_{T}-\int_{0}^{S_{N}}\left(1-\frac{\beta K}{N} \sum_{n=1}^{m} \frac{1}{K-n \Delta}\right) S_{T} f\left(S_{T}\right) \mathrm{d} S_{T} \\
= & (1-\beta) K \int_{-\infty}^{\ln S_{N}} f\left(\mathrm{e}^{y}\right) \mathrm{e}^{y} \mathrm{~d} y-\left(1-\frac{\beta K}{N} \sum_{n=1}^{m} \frac{1}{K-n \Delta}\right) \int_{-\infty}^{\ln S_{N}} \mathrm{e}^{y} f\left(\mathrm{e}^{y}\right) \mathrm{e}^{y} \mathrm{~d} y \\
= & (1-\beta) K \frac{1}{\sqrt{2 \pi} \sigma} \int_{-\infty}^{\ln S_{N}} \mathrm{e}^{-\frac{1}{2} \frac{(y-\mu)^{2}}{\sigma^{2}}} \mathrm{~d} y \\
& -\left(1-\frac{\beta K}{N} \sum_{n=1}^{m} \frac{1}{K-n \Delta}\right) \frac{1}{\sqrt{2 \pi} \sigma} \int_{-\infty}^{\ln S_{N}} \mathrm{e}^{y} \mathrm{e}^{-\frac{1}{2} \frac{(y-\mu)^{2}}{\sigma^{2}}} \mathrm{~d} y
\end{aligned}
$$




$$
\begin{aligned}
= & (1-\beta) K \frac{1}{\sqrt{2 \pi}} \int_{-\infty}^{\frac{\ln S_{N}-\mu}{\sigma}} \mathrm{e}^{-\frac{1}{2} z^{2}} \mathrm{~d} z \\
& -\left(1-\frac{\beta K}{N} \sum_{n=1}^{m} \frac{1}{K-n \Delta}\right) \frac{1}{\sqrt{2 \pi}} \int_{-\infty}^{\frac{\ln S_{N}-\mu}{\sigma}} \mathrm{e}^{\mu+z \sigma} \mathrm{e}^{-\frac{1}{2} z^{2}} \mathrm{~d} z \\
= & (1-\beta) K \frac{1}{\sqrt{2 \pi}} \int_{-\infty}^{\frac{\ln S_{N}-\mu}{\sigma}} \mathrm{e}^{-\frac{1}{2} z^{2}} \mathrm{~d} z \\
& -\left(1-\frac{\beta K}{N} \sum_{n=1}^{m} \frac{1}{K-n \Delta}\right) \frac{1}{\sqrt{2 \pi}} \int_{-\infty}^{\frac{\ln S_{N}-\mu}{\sigma}} \mathrm{e}^{\mu+z \sigma} \mathrm{e}^{-\frac{1}{2}(z-\sigma)^{2}} \mathrm{~d} z \\
= & (1-\beta) K N\left(\frac{\ln S_{N}-\mu}{\sigma}\right) \\
& -\left(1-\frac{\beta K}{N} \sum_{n=1}^{m} \frac{1}{K-n \Delta}\right) N\left(\frac{\ln S_{N}-\mu-\sigma^{2}}{\sigma}\right)
\end{aligned}
$$

and

$$
\begin{aligned}
& I_{2}=\int_{S_{m+1}}^{S_{m}}\left[\left(1-\frac{\beta m}{N}\right) K-S_{T}+\frac{\beta S_{T} K}{N} \sum_{n=1}^{m} \frac{1}{K-n \Delta}\right] f\left(S_{T}\right) \mathrm{d} S_{T} \\
& =\left(1-\frac{\beta m}{N}\right) K \int_{S_{m+1}}^{S_{m}} f\left(\mathrm{e}^{y}\right) \mathrm{e}^{y} \mathrm{~d} y-\left(1-\frac{\beta K}{N} \sum_{n=1}^{m} \frac{1}{K-n \Delta}\right) \int_{S_{m+1}}^{S_{m}} \mathrm{e}^{y} f\left(\mathrm{e}^{y}\right) \mathrm{e}^{y} \mathrm{~d} y \\
& =\left(1-\frac{\beta m}{N}\right) K \frac{1}{\sqrt{2 \pi} \sigma} \int_{S_{m+1}}^{S_{m}} \mathrm{e}^{-\frac{1}{2} \frac{(y-\mu)^{2}}{\sigma^{2}}} \mathrm{~d} y \\
& -\left(1-\frac{\beta K}{N} \sum_{n=1}^{m} \frac{1}{K-n \Delta}\right) \frac{1}{\sqrt{2 \pi} \sigma} \int_{S_{m+1}}^{S_{m}} \mathrm{e}^{y} \mathrm{e}^{-\frac{1}{2} \frac{(y-\mu)^{2}}{\sigma^{2}}} \mathrm{~d} y \\
& =\left(1-\frac{\beta m}{N}\right) K \frac{1}{\sqrt{2 \pi}} \int_{\frac{S_{m+1}-\mu}{\sigma}}^{\frac{S_{m}-\mu}{\sigma}} \mathrm{e}^{-\frac{1}{2} z^{2}} \mathrm{~d} z \\
& -\left(1-\frac{\beta K}{N} \sum_{n=1}^{m} \frac{1}{K-n \Delta}\right) \frac{1}{\sqrt{2 \pi}} \int_{\frac{S_{m+1}-\mu}{\sigma}}^{\frac{S_{m}-\mu}{\sigma}} \mathrm{e}^{\mu+\frac{1}{2} \sigma^{2}} \mathrm{e}^{-\frac{1}{2}(z-\sigma)^{2}} \mathrm{~d} z \\
& =\left(1-\frac{\beta m}{N}\right) K\left[N\left(\frac{\ln S_{m}-\mu}{\sigma}\right)-N\left(\frac{\ln S_{m+1}-\mu}{\sigma}\right)\right] \\
& -\left(1-\frac{\beta K}{N} \sum_{n=1}^{m} \frac{1}{K-n \Delta}\right)\left[N\left(\frac{\ln S_{m}-\mu-\sigma^{2}}{\sigma}\right)-N\left(\frac{\ln S_{m+1}-\mu-\sigma^{2}}{\sigma}\right)\right]
\end{aligned}
$$

Similarly,

$$
\begin{aligned}
I_{3}= & \int_{S_{1}}^{K}\left(K-S_{T}\right) f\left(S_{T}\right) \mathrm{d} S_{T} \\
= & K \int_{\ln S_{1}}^{\ln K} f\left(\mathrm{e}^{y}\right) \mathrm{e}^{y} \mathrm{~d} y-\int_{\ln S_{1}}^{\ln K} \mathrm{e}^{y} f\left(\mathrm{e}^{y}\right) \mathrm{e}^{y} \mathrm{~d} y \\
= & K \frac{1}{\sqrt{2 \pi} \sigma} \int_{\ln S_{1}}^{\ln K} \mathrm{e}^{\frac{(y-\mu)^{2}}{\sigma^{2}}} \mathrm{~d} y-\frac{1}{\sqrt{2 \pi} \sigma} \int_{\ln S_{1}}^{\ln K} \mathrm{e}^{y} \mathrm{e}^{\frac{(y-\mu)^{2}}{\sigma^{2}}} \mathrm{~d} y \\
= & K\left[N\left(\frac{\ln K-\mu}{\sigma}\right)-N\left(\frac{\ln S_{1}-\mu}{\sigma}\right)\right] \\
& -\mathrm{e}^{\mu+\frac{1}{2} \sigma^{2}}\left[N\left(\frac{\ln K-\mu-\sigma^{2}}{\sigma}\right)-N\left(\frac{\ln S_{1}-\mu-\sigma^{2}}{\sigma}\right)\right]
\end{aligned}
$$




\subsection{Option Price under Stochastic Interest Rates}

\subsubsection{Vasicek Model}

The price of Put Option $P$ with the underlying stock price dynamic and interest rate following the Vasicek model reads

$$
P=P(0, T) E^{T}\left[V_{T}\right]
$$

where $P(0, T)$ is the zero-coupon bond price (2.16) with $\mu_{T}, \sigma_{T}^{2}$ the mean and variance under $T$-forward measure. Thus,

$$
\begin{aligned}
P= & P(0, T) E^{T}\left[V_{T}\right] \\
= & P(0, T) K(1-\beta) N\left(d_{1}\right)-P(0, T)\left(1-\frac{\beta K}{N} \sum_{n=1}^{m} \frac{1}{K-n \Delta}\right) N\left(d_{2}\right) \\
& +P(0, T)\left(1-\frac{\beta m}{N}\right) K\left[N\left(d_{3}\right)-N\left(d_{4}\right)\right] \\
& -P(0, T)\left(1-\frac{\beta K}{N} \sum_{n=1}^{m} \frac{1}{K-n \Delta}\right)\left[N\left(d_{5}\right)-N\left(d_{6}\right)\right] \\
& +P(0, T)\left[N\left(d_{7}\right)-N\left(d_{8}\right)\right]-P(0, T) \mathrm{e}^{\mu+\frac{1}{2} \sigma^{2}}\left[N\left(d_{9}\right)-N\left(d_{10}\right)\right]
\end{aligned}
$$

where,

$$
\begin{array}{ll}
d_{1}=\frac{\ln S_{N}-\mu_{T}}{\sigma_{T}} & d_{6}=\frac{\ln S_{m+1}-\mu_{T}-\sigma_{T}^{2}}{\sigma_{T}} \\
d_{2}=\frac{\ln S_{N}-\mu_{T}-\sigma_{T}^{2}}{\sigma_{T}} & d_{7}=\frac{\ln K-\mu_{T}}{\sigma_{T}} \\
d_{3}=\frac{\ln S_{m}-\mu_{T}}{\sigma_{T}} & d_{8}=\frac{\ln S_{1}-\mu_{T}}{\sigma_{T}} \\
d_{4}=\frac{\ln S_{m+1}-\mu_{T}}{\sigma_{T}} & d_{9}=\frac{\ln K-\mu_{T}-\sigma_{T}^{2}}{\sigma_{T}} \\
d_{5}=\frac{\ln S_{m}-\mu_{T}-\sigma_{T}^{2}}{\sigma_{T}} & d_{10}=\frac{\ln S_{1}-\mu_{T}-\sigma_{T}^{2}}{\sigma_{T}}
\end{array}
$$

and

$$
\begin{aligned}
\mu_{T} & =\ln S_{0}-\frac{\sigma_{1}^{2}}{2} T-r_{0} \frac{1-\mathrm{e}^{-a T}}{a}+\left(\frac{\theta}{a}-\frac{\sigma_{2}^{2}}{a^{2}}\right)\left[T-\frac{1-\mathrm{e}^{-a T}}{a}\right]+\frac{\sigma_{2}^{2}}{2 a} \frac{1-\mathrm{e}^{-a T}}{a} \\
\sigma_{T}^{2} & =\frac{\sigma_{2}^{2}}{a^{2}}\left[T-2 \frac{1-\mathrm{e}^{-a T}}{a}+\frac{1-\mathrm{e}^{-2 a T}}{2 a}\right]+\sigma_{1}^{2} T .
\end{aligned}
$$

\subsubsection{Hull-White Model}

The Put Option price under the extended Vasicek, Hull-White model is expressed by (3.12) where the mean $\mu_{T}$ and variance $\sigma_{T}^{2}$ under the $T$-forward measure are

$$
\begin{aligned}
\mu_{T}= & \ln S_{0}-\frac{\sigma_{1}^{2}}{2} T+r_{0} \frac{1-\mathrm{e}^{-a T}}{a}-\frac{\sigma_{2}^{2}}{a}\left[\frac{\mathrm{e}^{-2 a T}\left((2 a T-3) \mathrm{e}^{2 a T}+4 \mathrm{e}^{a T}-1\right)}{2 a^{2}}\right] \\
& +\int_{0}^{T} \mathrm{e}^{-a t} \int_{0}^{t} \theta(s) \mathrm{e}^{a s} \mathrm{~d} s \mathrm{~d} t \\
\sigma_{T}^{2}= & \frac{\sigma_{2}^{2}}{a^{2}}\left[T-2 \frac{1-\mathrm{e}^{-a T}}{a}+\frac{1-\mathrm{e}^{-2 a T}}{2 a}\right]+\sigma_{1}^{2} T
\end{aligned}
$$


where the zero-coupon bond price is

$$
P(0, T)=\mathrm{e}^{\frac{r_{0}\left(\mathrm{e}^{-a T}-1\right)}{a}-\int_{0}^{T} \mathrm{e}^{-a s} \int_{0}^{s} \theta\left(\mathrm{e}^{a u}\right) \mathrm{d} u \mathrm{ds}+\frac{\sigma_{2}^{2}}{2 a^{2}}\left[T+\frac{1-\mathrm{e}^{-2 a T}}{2 a}-\frac{2}{a}\left(1-\mathrm{e}^{-a T}\right)\right]} .
$$

\section{Conclusion}

The findings in this paper show the existence of the closed form solutions for Call and Put Option Pricing under the linear investment strategy and are amenable to actual market implementation. The key benefit (unlike the classical Black-Scholes Model) is that not only such options are more realistic (i.e., stochastic interest rates and discrete time trading strategy), but in addition they command lower pricing (i.e., become more attractive from the investor's perspective) when compared to Black-Scholes pricing. The future research will focus on extension of the Option pricing model based on dynamic investment strategy to Jump-diffusion processes, which typically fits market data better than the regular diffusion processes.

\section{Conflicts of Interest}

The authors declare no conflicts of interest regarding the publication of this paper.

\section{References}

[1] Wang, X.F. and Wang, L. (2007) Study on Black-Scholes Stock Option Pricing Model Based on Investment Strategy. International Journal of Innovative Computing, Information and Control, 3, 1755-1780.

[2] Wang, X.F., Wang, L. and Zhai, A.M. (2007) Research on Black-Scholes Stock Put Option Model Based on Dynamic Investment Strategy. International Conference on Wireless Communications, Networking and Mobile Computing, Shanghai, 21-25 September 2007, 4128-4131. https://doi.org/10.1109/WICOM.2007.1020

[3] Zhang, X., Shu, H.S., Kan, X., Fang, Y.Y. and Zheng, Z.W. (2018) The Call Option Pricing Based on Investment Strategy with Stochastic Interest Rate. Journal of Mathematical Finance, 8, 43-57. https://doi.org/10.4236/jmf.2018.81004

[4] Ghorbani, N. and Korzeniowski, A. (2020) Adaptive Risk Hedging for Call Options under Cox-Ingersoll-Ross Interest Rates. Journal of Mathematical Finance, 10, 697704. https://doi.org/10.4236/jmf.2020.104040

[5] Korzeniowski, A. and Ghorbani, N. (2021) Put Options with Linear Investment for Hull-White Interest Rates. Journal of Mathematical Finance, 11, 152-162. https://doi.org/10.4236/jmf.2021.111007

[6] Li, M., Wang, X.F. and Sun, F.F. (2019) Pricing of Proactive Hedging European Option with Dynamic Discrete Strategy. Dynamics in Nature and Society, 2019, Article ID: 1070873 . https://doi.org/10.1155/2019/1070873

[7] Charandabi, S.E. and Kamyar K. (2021) Using A Feed Forward Neural Network Algorithm to Predict Prices of Multiple Cryptocurrencies. European Journal of Business Management and Research, 6, 15-19. https://doi.org/10.24018/ejbmr.2021.6.5.1056

[8] Charandabi, S.E. and Kamyar K. (2021) Prediction of Cryptocurrency Price Index 
Using Artificial Neural Networks: A Survey of the Literature. European Journal of Business Management and Research, 6, 17-20.

https://doi.org/10.24018/ejbmr.2021.6.6.1138

[9] Krause, T.A. (2019) Put-Call Parity in Equity Options Markets: Recent Evidence. Theoretical Economics Letters, 9, 563-569. https://doi.org/10.4236/tel.2019.94039

[10] Vasicek, O. (1977) An Equilibrium Characterization of the Term Structure. Journal of Financial Economics, 5, 177-188. https://doi.org/10.1016/0304-405X(77)90016-2 